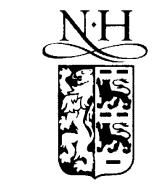

ELSEVIER

\title{
Feasibility and limitation of track studies using atomic force microscopy
}

\author{
D. Nikezic, J.P.Y. Ho, C.W.Y. Yip, V.S.Y. Koo, K.N. Yu * \\ Department of Physics and Materials Science, City University of Hong Kong, Tat Chee Avenue, Kowloon Tong, Kowloon, Hong Kong
}

Received 14 March 2002; received in revised form 17 July 2002

\begin{abstract}
Atomic force microscopy (AFM) has been employed to investigate characteristics of tracks of heavy charged particles in solid state nuclear track detectors (SSNTDs). In the present work, we have performed simulations of the track structures revealed by AFM based only on geometrical considerations of the tracks and two types of probes (the ultralever and the ultrahigh aspect ration probe). The purpose of this work is to determine the limitations and constraints of the AFM technique when it is applied to track investigations. The ultralever has comparable dimensions as the tracks in SSNTDs etched for a short time. In some cases, the ultralever is too large or its geometry does not match those of the tracks, so these tracks cannot be scanned properly. In most cases, the ultralever can measure the diameter of the tracks with a rather high precision, but measurements of the depths can be misleading if the track depths are larger than the length of the ultralever. The ultrahigh aspect ratio probe, with an aspect ratio better than 10:1, can record tracks with rather high accuracy if the track depths are not larger than probe length. The technique involving the mounting of nanotubes on AFM tips, which has become available in recent years, should be able to record almost perfect track profiles.
\end{abstract}

(C) 2002 Elsevier Science B.V. All rights reserved.

PACS: 29.40; 23.60

Keywords: Solid state nuclear track detector; Atomic force microscopy; LR115 detector; CR39 detector

\section{Introduction}

Fast heavy ions can produce latent tracks in some dielectric materials. After adequate treatment, such as chemical or electrochemical etching, these latent tracks can be made visible under the

\footnotetext{
${ }^{*}$ Corresponding author. Tel.: +852-27887812; fax: $+852-$ 27887830.

E-mail address: peter.yu@cityu.edu.hk (K.N. Yu).
}

optical microscope. This is the operational principle for solid state nuclear track detectors (SSNTDs). The technique has been extensively investigated in the literature, and has been widely applied in many fields of science and technology.

Tracks in SSNTDs have been studied with the optical microscope for a long time. Recently, some researchers have applied the atomic force microscope (AFM) to study the tracks in SSNTDs. The following are the examples. He et al. [1] employed the AFM to measure the bulk etch rate of CR39 
detector. Yamamoto et al. [2,3] also used the AFM to determine track parameters and the sensitivity function $s=V_{\mathrm{t}} / V_{\mathrm{b}}$ of the CR39 detector for heavy ions, where $V_{\mathrm{t}}$ is the track etch rate and $V_{\mathrm{b}}$ is the bulk etch rate. The bulk etch rate of the CR39 detector was measured with AFM by using the "mask method" by Yasuda et al. [4]. Surface roughness and the function $s$ of different samples of CR39 detector were studied with AFM by Vazquez-Lopez et al. [5]. AFM was also used to investigate the tracks of nuclear reaction products in CR39 detector [6]. Yasuda et al. [7] used AFM to estimate the latent track size in CR39 after irradiation with $\mathrm{C}$ and $\mathrm{Fe}$ ions. Palmino et al. [8] used AFM to study the real-time evolution of tracks in the LR115 detector under chemical etching.

However, despite the growing interest in using the AFM to study tracks in SSNTDs, the feasibility or limitations have not been explored or studied in detail. In the present work, we have performed computer simulations of track measurements with the AFM in order to show the feasibility or limitations of the technique based on geometry alone.

\section{Atomic force microscopy and general consider- ations}

The AFM system we use in the present investigation is the Autoprobe $\mathrm{CP}$ model from Park Scientific Instruments (1171 Borregas Avenue, Sunnyvale, CA 94089). The probe of the AFM can be a cantilever (Fig. 1(a)), ultralever (Fig. 1(b)) or ultrahigh aspect ratio probe (Fig. 1(c)), the first two being available in our own laboratory. The main differences among these probes are the shapes, with the ultralever being sharper than the cantilever, and the ultrahigh aspect ratio probe sharper than the ultralever. When the probe scans across a surface, a profile of the scanned surface is captured digitally through computerization, which can be shown on the monitor of instrument or stored for further analyses. The problem with the cantilever lies with the shape of the tip: the length (only 0.2 $\mu \mathrm{m})$ is too short and the opening angle $\left(18^{\circ}\right)$ is too large. The shape of the cantilever imposes strong limitations when the tracks are scanned. On the contrary, the ultralever has a much smaller opening angle of $10^{\circ}$ and a larger length of $4 \mu \mathrm{m}$. Although the ultralever is more convenient for track studies, it is still not a perfect probe and will still impose some limitations in track studies. However, this is already the sharpest probe available in our laboratory. After gaining some experience of track studies with the AFM, we concluded that ultralever is more convenient than the cantilever for studying tracks. The discussions below will focus on the ultralever to provide a base for comparison between the simulated and experimental results in the future. Nevertheless, some simulations will also be carried out for the ultrahigh aspect ratio probe, although it is not available in our laboratory, to demonstrate its advantages.

In the course of a measurement, the ultralever moves across the investigated surface and records its profile. We simulated the situations when the (a)

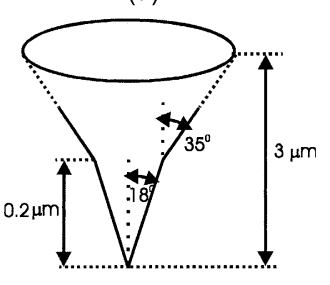

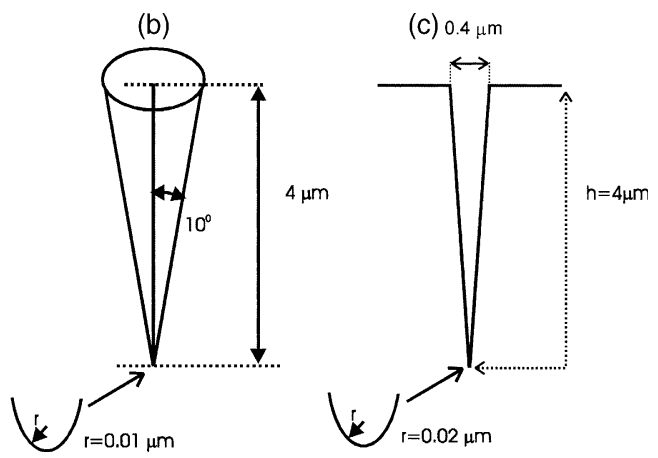

Fig. 1. Geometry of the cantilever (a), the ultralever (b) and the ultrahigh aspect ratio probe (c). 


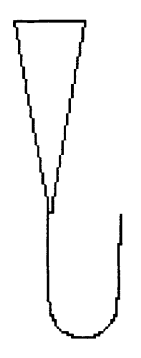

(a)

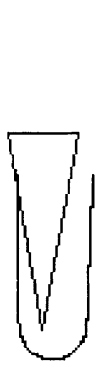

(b)

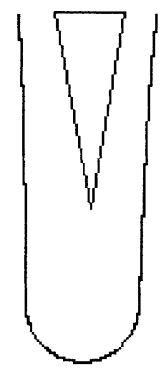

(c) (d)

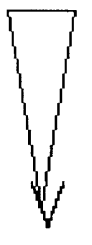

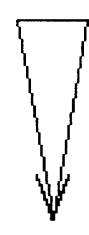

(e)
Fig. 2. Typical situations when the ultralever passed across a typical track for the LR115 detector (a)-(c) and a typical track for the CR39 detector (d)-(e).

ultralever passed across the track. A number of typical situations are presented in Fig. 2(a)-(e). In Fig. 2(a)-(c), deep tracks with steep walls, which are typical for the LR 115 detector, are shown with the ultralever in different positions. In Fig. 2(a), the ultralever has just come into the track and started to slide past the edge of the track. In Fig. 2(b), the ultralever has already penetrated deep into the track, but its tip has lost contact with the track wall with the track edge supporting the lateral side of the ultralever. Fig. 2(c) shows the case where the ultralever is shorter than the depth of the track and cannot record the profile of the bottom. In this case, the ultralever will move horizontally until its lateral side touches the opposite wall of the track again. Fig. 2(d) and (e) show passage of the ultralever across a typical track in the CR39 detector. Being not so steep, the tracks in the CR39 detector are much more convenient to study with AFM. As presented in Fig. 1, the radius of curvature of the tip is $0.01 \mu \mathrm{m}$. This feature will disable studies of the very bottom points of tracks and, in some cases, influence on the recorded track profiles.

In addition to the previous considerations, we should also note that the tracks and the ultralever are not two-dimensional objects as shown in Fig. 2. In fact, they are three-dimensional structures and special attention should be paid to the path through which the ultralever passes through the track. Fig. 3 shows three such paths across the track. Path 1 is on the periphery of the track and the recorded profile is the line $1^{\prime}$, which is rather

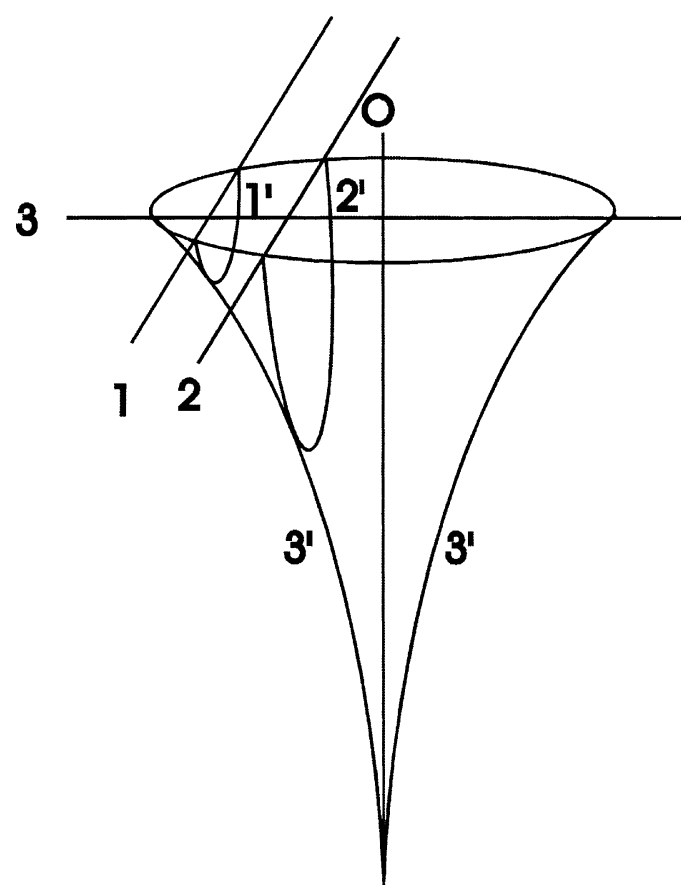

Fig. 3. Three paths through which the ultralever passes through a track.

shallow. Path 2 is close to the center and the recorded profile $2^{\prime}$ is much deeper than $1^{\prime}$. Finally, path 3 is directly crossing the center $\mathrm{O}$ of the track. The recorded profile is denoted with $3^{\prime}$. As expected, only this path can give a depth close to the real track depth.

During measurements, the ultralever scans the studied surface many times, typically with a $256 \times 256$ resolution for a scanning area of $25 \times$ $25 \mu^{2}$. In other words, the typical distance between neighboring paths is about $0.1 \mu \mathrm{m}$. The track diameter for a short etching time, which will be used in the present study, is less than $1 \mu \mathrm{m}$. For example, the diameter of the alpha-particle track with $5 \mathrm{MeV}$ incident energy in the CR39 detector etched for $15 \mathrm{~min}$ is about $0.3 \mu \mathrm{m}$. Under such a condition, the ultralever only passes across the track a few times, which can give erroneous results for the track parameters. In such cases, better resolution is needed, i.e. a smaller area should be scanned while keeping the $256 \times 256$ resolution. However, the probability to find tracks in a smaller scanned area is also smaller. 


\section{Computational simulation}

Here we perform computational simulations of the track structures revealed by the AFM. For simplicity, we assume that the scanning resolution is sufficiently good so that the probes always cross the track centers. The tracks of alpha particles in CR39 and LR115 detectors were plotted by previously described computer programs [9]. Details of these programs will not be repeated here. Each track is represented by a set of coordinates for the track wall and stored in the memory of the computer. During the simulations, the AFM probe moves step by step across the track in such a way that it touches the track wall, only at one point. For a given position in the horizontal direction ( $x$ axis), the computer calculates a feasible position of the probe in the vertical direction ( $y$-axis) based on geometrical considerations. Subsequent positions of the tip, when the probe travels across the track, are stored in the computer memory, and they represent the profile recorded by the AFM. The computer program was written in standard Fortran90, and graphical on-line presentation of the scanning process is enabled on the computer screen.

\section{Results}

Simulations have been performed for the ultralever for LR115 and CR39 detectors for alphaparticle energies between 1 and $4 \mathrm{MeV}$ for LR115, and between 1 and $5 \mathrm{MeV}$ for CR39. In all cases the incident angle was taken to be $90^{\circ}$. The bulk etch rates were assumed to be 1 and $3.27 \mu \mathrm{m} \mathrm{h}^{-1}$ for CR39 and LR115, respectively, in the simulations. The results are shown in Figs. 4-7. For each alpha-particle energy, there will be a pair of curves. The calculated track shape is given on the left while the simulated profile recorded by AFM is given on the right. The scale for $1 \mu \mathrm{m}$ is also given for evaluation for the track dimension.

Fig. 4 gives the results for the CR39 detectors which have been etched for $15 \mathrm{~min}$. The recorded profiles are very similar to the calculated ones, but the depths are always smaller, with the difference between the simulated and calculated depths being larger for smaller energies. The results for an etching time of $30 \mathrm{~min}$ are shown in Fig. 5. All tracks are in the sharp developing phase. The opening angles of the track tips are larger than the opening angle of the AFM ultralever so this kind of tracks can be successfully scanned with the

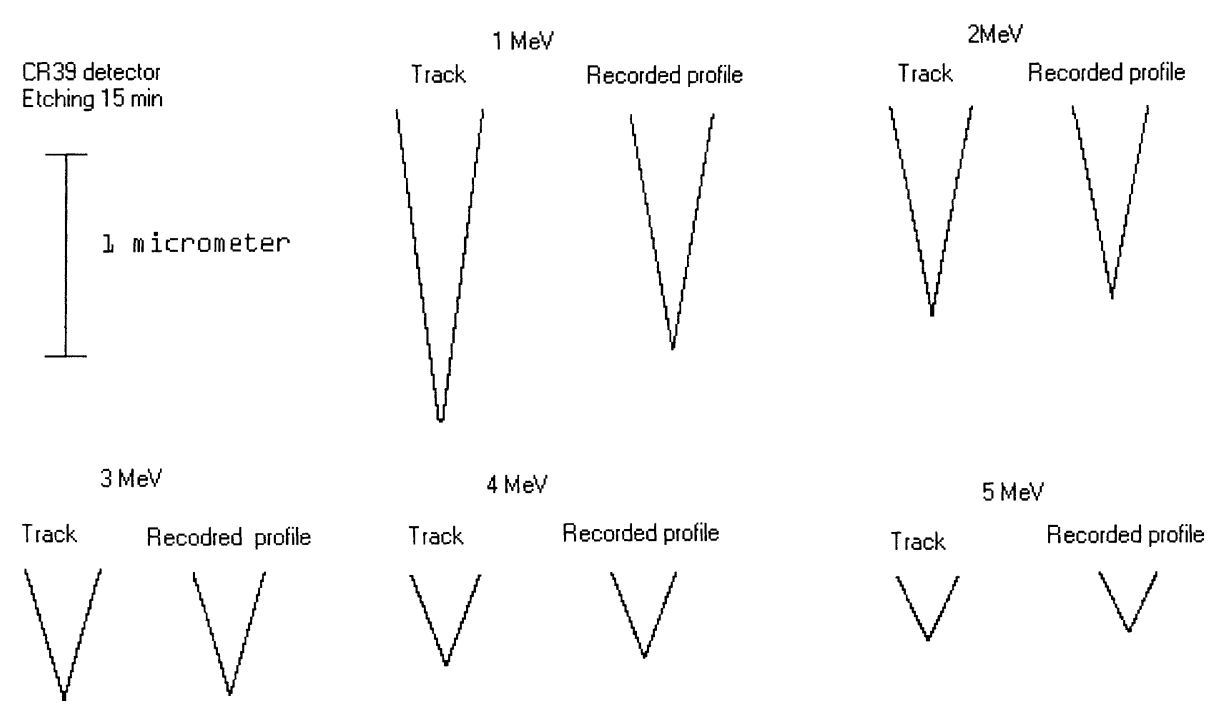

Fig. 4. Calculated track shape (left) and simulated track shape recorded by the ultralever of the AFM (right) for different alpha energies, in CR39 etched for $15 \mathrm{~min}$. 


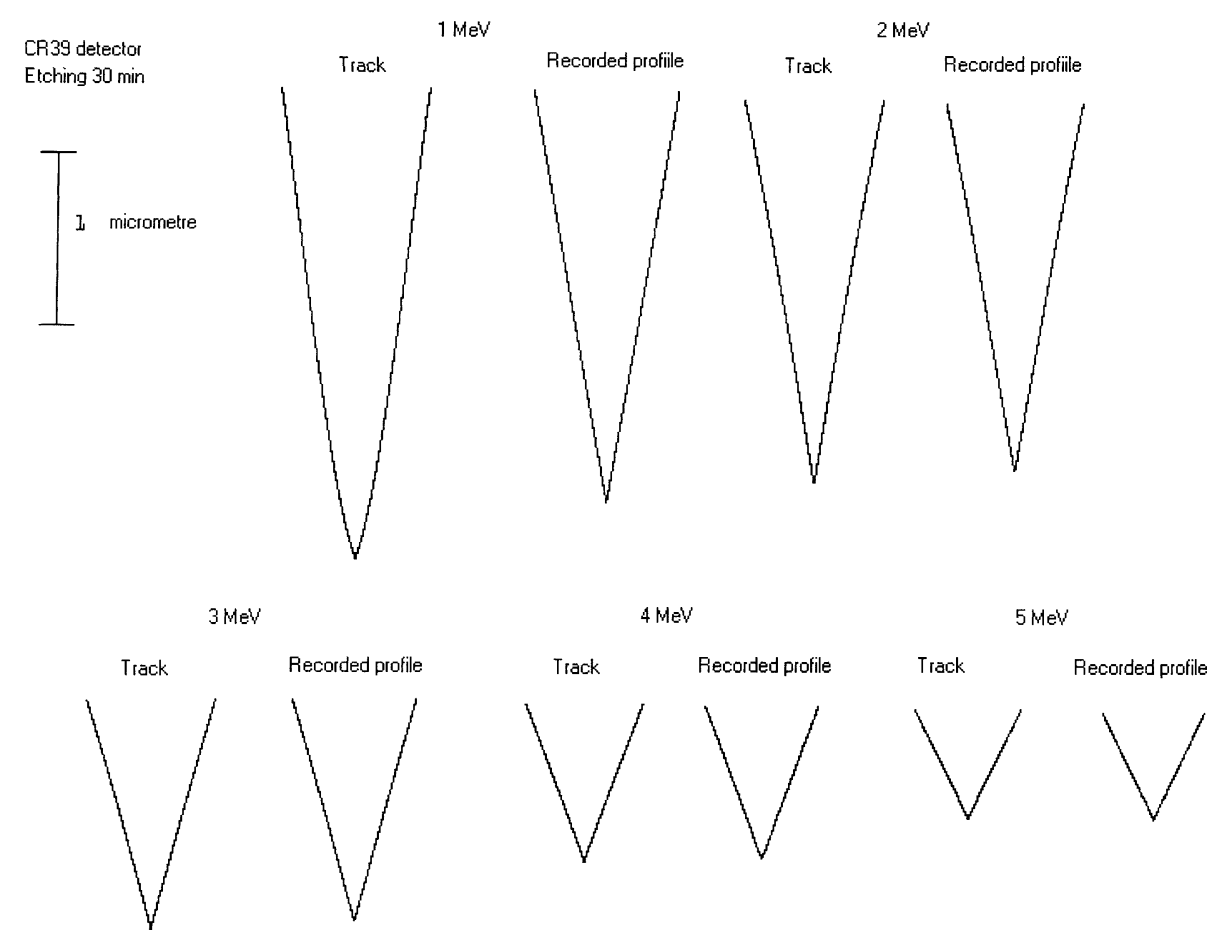

Fig. 5. Calculated track shape (left) and simulated track shape recorded by the ultralever of the AFM (right) for different alpha energies, in CR39 etched for $30 \mathrm{~min}$.

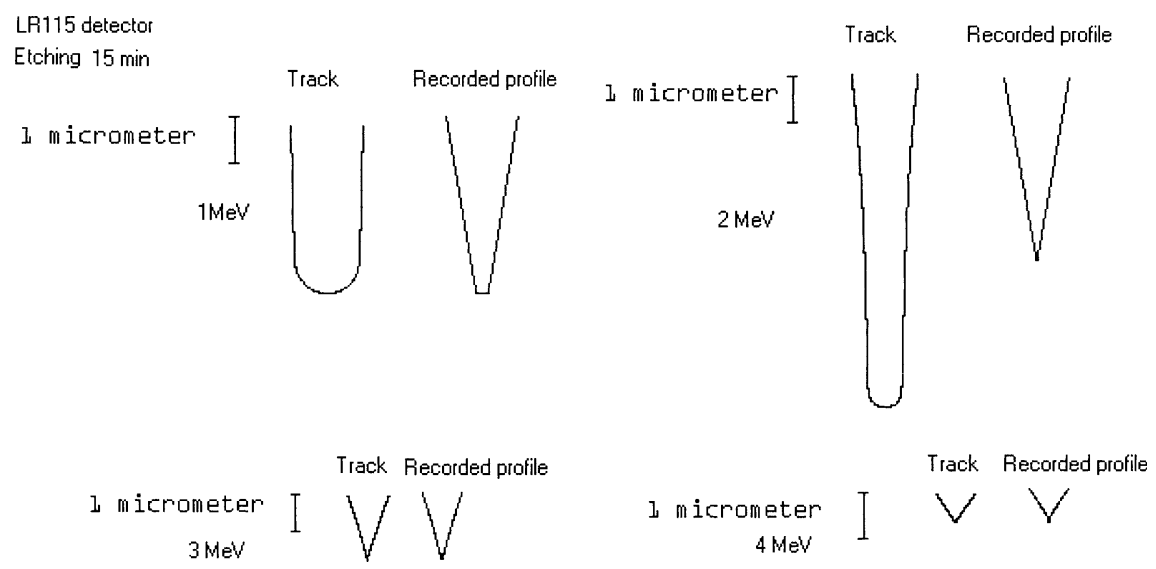

Fig. 6. Calculated track shape (left) and simulated track shape recorded by the ultralever of the AFM (right) for different alpha energies, in LR115 etched for $15 \mathrm{~min}$.

AFM. However, the recorded depths are smaller than the real ones and, as in the previous case, the differences are more pronounced for smaller energies. In the low-energy region, the difference can be significant. The depth of the tracks from alpha particles with energies larger than $3 \mathrm{MeV}$ can be accurately measured with AFM.

The results for LR115 detectors are shown in Fig. 6 for an etching time of $15 \mathrm{~min}$. The appearances for the LR115 detectors are very different 


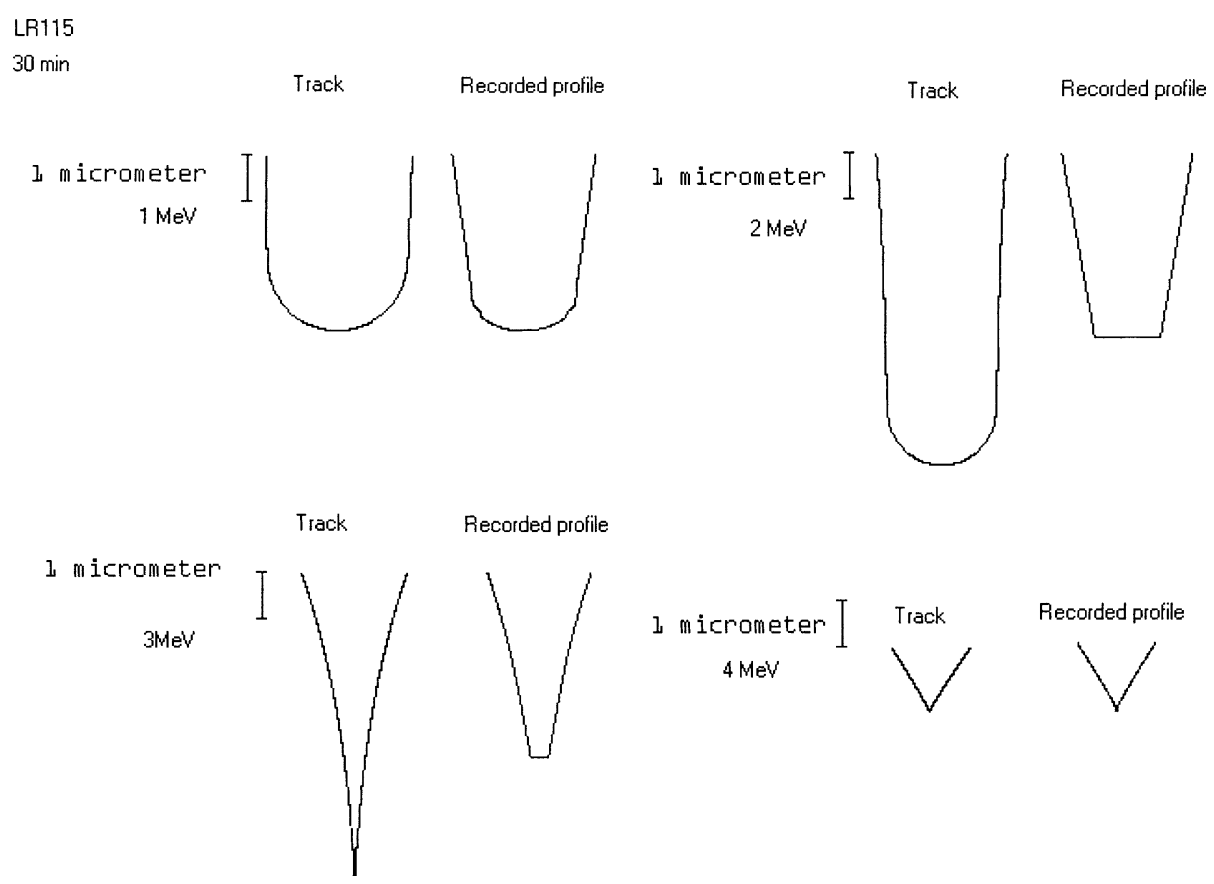

Fig. 7. Calculated track shape (left) and simulated track shape recorded by the ultralever of the AFM (right) for different alpha energies, in LR115 etched for $30 \mathrm{~min}$.

from those for the CR39 detectors. Due to the very fast etching along the particle track in the low energy region (Bragg peak), very deep tracks are created with almost vertical walls. This is an inconvenient situation for AFM and the track profiles recorded by AFM can suffer severe distortions. The tracks for alpha energies of 3 and $4 \mathrm{MeV}$ are not so steep and AFM can scan them more properly. Fig. 7 shows the results for an etching time of $30 \mathrm{~min}$. The simulated track for the alpha energy of $1 \mathrm{MeV}$ particle now has a rounded tip and the simulated depth is almost correct, but there are still some distortions of the track wall as recorded by the AFM. The calculated track for an alpha energy of $2 \mathrm{MeV}$ is very deep in the detector. Since the track is too deep and the ultralever cannot penetrate to the bottom, the depth measured by the AFM will not be correct. The situation is similar for the track of an alpha energy of $3 \mathrm{MeV}$. On the contrary, the track for an alpha energy of $4 \mathrm{MeV}$ is convenient and suitable for AFM scanning which will give a proper profile.

Table 1 summarizes the track depths calculated by the computer program and from the AFM simulations, and the column "\%" gives the percentage difference between the two. It can be seen that the error can be as large as $50 \%$.

As mentioned above, we will also carry out some simulations for the ultrahigh aspect ratio probe, although it is not available in our laboratory, to demonstrate its advantages. In fact, different probes are available on the market (e.g. see the webpages: www.topometrix.com or www. spmprobes.com). As an illustration, we simulated the most inconvenient case identified from the above results, i.e. tracks for LR 115 detectors from $2 \mathrm{MeV}$ alpha particles. According to the manufacturer, the length is $4 \mu \mathrm{m}$ and the aspect ratio is 10:1. The results are shown in Fig. 8. In the case shown in Fig. 8(a), the probe is shorter than the track depth so it cannot reach the bottom of the track, and a flattened bottom of the recorded object appears again. The track wall, although nearly vertical, will be almost perfectly recorded. Fig. 8(b) shows the results for a track formed after etching for $5 \mathrm{~min}$ in the LR 115 detector. Here, the track depth is shorter than the probe and the entire wall profile is accurately recorded. 
Table 1

Calculated track depths (under the columns "Track") and the simulated track depths recorded by ultralever of AFM (under the columns "AFM") for CR39 and LR115 detectors for different alpha energies and the percentage difference (under the columns " $\%$ ")

\begin{tabular}{|c|c|c|c|c|c|c|c|c|c|c|c|c|}
\hline \multirow{3}{*}{$\begin{array}{l}\text { Energy } \\
(\mathrm{MeV})\end{array}$} & \multicolumn{6}{|c|}{ Depth in CR39 $(\mu \mathrm{m})$} & \multicolumn{6}{|c|}{ Depth in LR115 $(\mu \mathrm{m})$} \\
\hline & \multicolumn{3}{|c|}{$15 \mathrm{~min}$} & \multicolumn{3}{|l|}{$30 \mathrm{~min}$} & \multicolumn{3}{|l|}{$15 \mathrm{~min}$} & \multicolumn{3}{|l|}{$30 \mathrm{~min}$} \\
\hline & Track & AFM & $\%$ & Track & AFM & $\%$ & Track & AFM & $\%$ & Track & AFM & $\%$ \\
\hline 1 & 1.62 & 1.17 & 28 & 2.77 & 2.4 & 13 & 3.79 & 3.79 & 0 & 3.79 & 3.79 & 0 \\
\hline 2 & 1.04 & 0.97 & 7 & 2.26 & 2.14 & 5 & 7.12 & 3.89 & 45 & 7.12 & 4 & 43 \\
\hline 3 & 0.65 & 0.6 & 8 & 1.33 & 1.28 & 4 & 1.77 & 1.67 & 5 & 6.57 & 4 & 40 \\
\hline 4 & 0.45 & 0.41 & 9 & 0.92 & 0.88 & 4 & 0.63 & 0.61 & 3 & 1.34 & 1.34 & 0 \\
\hline 5 & 0.32 & 0.29 & 9 & 0.64 & 0.61 & 5 & - & - & - & - & - & - \\
\hline
\end{tabular}

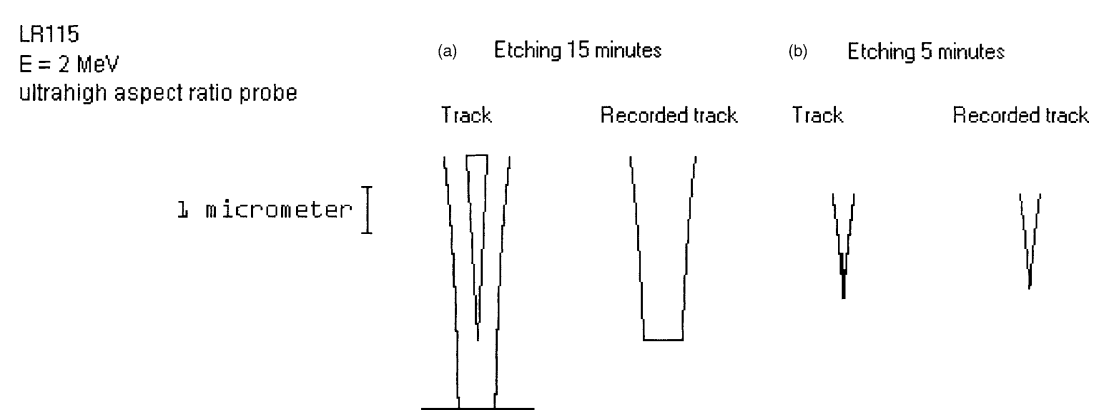

Fig. 8. Calculated track shape (left) and simulated track shape (right) recorded by the ultra high-aspect-ratio (10:1) probe, in LR115 detector etched 5 and $15 \mathrm{~min}$, for an incident alpha energy $2 \mathrm{MeV}$.

\section{Conclusions and discussion}

Computational simulations of track scanning in LR115 and CR39 detectors with the AFM have been performed. The computations in the present paper are only based on geometrical considerations of the probes (an ultralever and an ultrahigh aspect ration probe) and the tracks. Other limitations, such as the inertia of the ultralever, have not been taken into account.

The results presented in this paper show that the ultralever of the atomic force has limitations in track studies particularly for deep tracks with very steep walls, which are more frequently encountered in LR 115 detectors. In some cases the error in the track depths can be as large as $50 \%$. The recorded track profiles can also be wrong. CR39 detectors are more convenient and suitable to be studied with the AFM. The maximum discrepancy from measurements of track depths in CR39 is about 28\% (for incident alpha energy of $1 \mathrm{MeV}$ ).
On the other hand, probes with ultra high aspect ratios, e.g. better than 10:1, can record wall profiles very accurately, even for very steep tracks in the LR115 detectors. Problems still remain if the probe is shorter than the track length. To be able to study the full track profile, we need shallower tracks and thus shorter etching time.

The technique to mount nanotubes on AFM tips has become available a few years ago [10], for which the non-contact tapping mode of operation has been used. Simulation of AFM images of Si surfaces recorded using nanotubes were carried out [11], where chemical interaction between the tip of nanotube and the investigated surface was dominant over van der Waals forces. Applications of nanotubes have enabled recording of surface images on the atomic scale. To the best of our knowledge, there have not been attempts until now to employ nanotubes for track studies. Such investigations should be able to record almost perfect track profiles, and thus be able to give much 
better track etch rates $V_{\mathrm{t}}$ along the tracks, which are related to the restricted energy loss of particles in matter [12].

\section{Acknowledgements}

The present research is supported by the CERG grant CityU1081/01P from the Research Grant Council of Hong Kong (City University of Hong Kong reference number 9040639).

\section{References}

[1] Y.D. He, C.I. Hancox, M. Solarz, Nucl. Instr. and Meth. B 132 (1997) 109.

[2] M. Yamamoto, N. Yasuda, Y. Kaizuka, M. Yamagishi, T. Kanai, N. Ishigure, A. Furakawa, M. Kurano, N. Miyhara, M. Nakazawa, T. Doke, K. Ogura, Radiat. Meas. 28 (1997) 227.
[3] M. Yamamoto, N. Yasuda, M. Kurano, T. Kanai, A. Furakawa, N. Ishigure, K. Ogura, Nucl. Instr. and Meth. B 152 (1999) 349.

[4] N. Yasuda, M. Yamamoto, N. Miyhara, N. Ishigure, T. Kanai, K. Ogura, Nucl. Instr. and Meth. B 142 (1998) 111.

[5] C. Vazquez-Lopez, R. Fragaso, J.I. Golzarri, F. CastilloMejia, M. Fujii, G. Espinosa, Radiat. Meas. 34 (2001) 189.

[6] N. Rozlosnik, C.S. Glavak, J. Palfalvi, L. Sojo-Bohus, C. Birattari, Radiat. Meas. 28 (1997) 277.

[7] N. Yasuda, K. Uchikawa, K. Amemiya, N. Watanabe, H. Takahashi, M. Nakazawa, M. Yamamoto, K. Ogura, Radiat. Meas. 34 (2001) 45.

[8] F. Palmino, D. Klein, J.C. Labrune, Radiat. Meas. 31 (1999) 209.

[9] D. Nikezic, K.N. Yu, Nucl. Instr. and Meth. B 196 (2002) 105.

[10] H. Dai, J.H. Hafner, A.G. Rinzler, D.T. Colbert, R.E. Smalley, Nature 384 (1996) 147.

[11] K. Tagami, N. Sasaki, M. Tsukada, Appl. Surf. Sci. 172 (2001) 301.

[12] B. Dörschel, D. Hermsdorf, K. Kadner, S. Starke, Radiat. Meas. 35 (2002) 177. 